\title{
Preparation and Characterisation of Niacin Nanoparticles by Desolvation Technique
}

\author{
Huda Syed and Abbaraju Krishna Sailaja* \\ RBVRR Women's College of Pharmacy, Osmania University, Barkatpura, Hyderabad, India
}

*Corresponding Author: Abbaraju Krishna Sailaja, RBVRR Women's College of Pharmacy, Osmania University, Barkatpura, Hyderabad, India.

Received: May 21, 2019; Published: September 05, 2019

DOI: 10.31080/ASPS.2019.03.0389

\begin{abstract}
The objective of the present work is to prepare niacin nanoparticles by desolvation technique using acetone as desolvating agent. Acetone was added continuously into 1\% BSA solution at pH 7 under stirring at 700rpm. In continuous addition method desolvating agent was added at a rate of $1 \mathrm{ml} / 1 \mathrm{~min}$. Appearance of turbidity was considered as end point. A cross linking agent was added to stabilize the nanoparticles and stirring was continued for 12 hours. The obtained nano formulations were studied for characterization and evaluation parameters. Drug content was found to be $75.4 \%$, Entrapment efficiency was found to be $75.77 \%$ and loading capacity was observed as $25.44 \%$
\end{abstract}

Keywords: Desolvation; Bovine Serum Albumin; Cross Linking Agent; Polymeric Materials

\section{Introduction \\ Definition}

Nanoparticles are sub -nanosized colloidal structures composed of synthetic or semi-synthetic polymer and the particle size ranges from 10-1000 $\mathrm{nm}$ in diameter. Nanospheres are solid core spherical particulates which are nanometric in size. They contain drug embedded within the matrix or adsorbed on to the surface. Nanocapsules are vesicular system in which drug is essentially encapsulated with in the central volume surrounded by an embryonic polymeric sheath. The selection of the appropriate method for the preparation of the nanoparticles depends on the physiochemical characteristics of the polymer and the drug to be loaded [1].

Niacin is a water-soluble vitamin belonging to the vitamin B family, which occurs in many animal and plant tissues. Niacin also known as nicotinic acid or vitamin B3 has been shown to positively regulate lipoprotein levels. It is one of the few compounds shown to significantly raise HDL levels and regress atherosclerosis. As such, niacin has become an attractive option for the treatment of dyslipidemia and atherosclerotic disease. Niacin or niacinamide is used for preventing vitamin B3 deficiency and related conditions such as pellagra. Interestingly, the flushing effect of niacin has been shown to be reduced by concomitant administration of NSAIDs as well as reformulated delivery systems that prolong or extend sys- temic niacin release. Medication and supplemental niacin are primarily used to treat cholesterol and pellagra (niacin deficiency). Insufficient niacin in the diet can cause nausea, skin and mouth lesions, anaemia, headaches, and tiredness $[2,3]$.

Desolvation, a thermodynamically driven self-assembly process for polymeric materials to prepare nanoparticles. The polymeric molecules form particles of different sizes depending on the preparation conditions such as protein content, $\mathrm{pH}$, ionic strength, concentration of cross linking agent, agitation speed, amount of desolvating agent etc. It contains three steps. 1. Protein dissolution, 2. Protein aggregation and 3. Protein de-aggregation. Desolvation de-aggregates the protein and turns the suspension colloidal and hence milky in appearance. Both lipophilic and hydrophilic drugs can be entrapped in nanoparticles using this technique [4].

Bovine serum albumin (BSA) was chosen as the polymer for the preparation of niacin nanoparticles. BSA has great potential as a nano-carrier in food and pharmaceutical applications. BSA is nontoxic and degradable in-vivo, so the nanoparticles generated by using it are easily adaptable to the human body [5].

The main purpose of this study was to prepare niacin nanoparticles using the desolvation technique using continuous addition of acetone as desolvating agent [6]. 


\section{Materials}

- Drug: Niacin.

- Polymer: BSA (Bovine serum albumin).

- Solvent: Acetone.

- Cross linking agent: Glutaraldehyde.

\section{Experimental Methodology}

Niacin nanoparticles were prepared by desolvation technique. BSA polymer was selected for this technique, Acetone was used as a solvent.

\section{Desolvation method}

Preparation of niacin nanoparticles was carried out by Desolvation technique. Desolvation is a thermodynamically driven self -assembly process for polymeric materials to prepare nanoparticles. Aqueous drug polymer dispersion was prepared and $\mathrm{pH}$ was adjusted to 7 (away from iso-electric point).The desolvating agent (Acetone) was added under continuous mechanical stirring. In continuous addition method the desolvating agent was added at a rate of $1 \mathrm{ml}$ per min. The appearance of turbidity was observed as the end point of the reaction. A cross-linking agent (glutaraldehyde $25 \%$ ) was added and stirring was continued. The solvent was removed by rotary evaporation at a vacuum pressure of $760 \mathrm{mmHg}$ Free flowing nanoparticles were obtained [6].

\section{Evaluation studies}

The obtained formulation of niacin nanoparticle by desolvation technique are evaluated for the following parameters.

\section{Determination of drug content}

Drug content was determined as follows, $50 \mathrm{mg}$ formulation was weighed accurately and transferred into $100 \mathrm{ml}$ beaker containing and dissolved in $50 \mathrm{ml}$ of methanol and kept for stirring at $600 \mathrm{rpm}$ for 3 hours respectively. The total amount of the drug in the nanoparticles was determined by UV spectrophotometrically at $262 \mathrm{~nm}$ [7].

\section{Scanning electron microscopy}

Size of the nanoparticles was determined by means of scanning electron microscope.

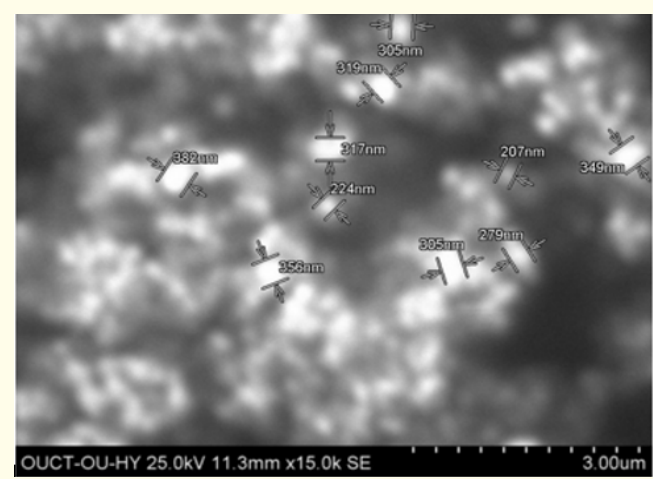

Figure 1

\section{Entrapment efficiency}

Entrapment efficiency indicates the amount of drug encapsulated in the formulation. $50 \mathrm{mg}$ of the prepared nanoparticles was dissolved in $40 \mathrm{ml}$ of $6.8 \mathrm{pH}$ phosphate buffer and was kept for ultracentrifuge at $17000 \mathrm{rpm}$ for $40 \mathrm{mins}$. Respectively [8].

Percentage entrapment efficiency may be calculated from the following formula:

Entrapment efficiency $=\frac{W-w}{W}$

\section{Loading capacity}

It indicates the capacity of the polymer to load a drug [9].

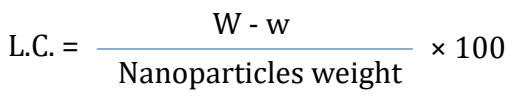

\section{In Vitro drug release kinetics}

In vitro drug release studies were conducted by means of orbitary shaker. $50 \mathrm{mg}$ of each accurately weighed formulation was transferred into $250 \mathrm{ml}$ conical flask containing $50 \mathrm{ml} \mathrm{pH} 7.4$ phosphate buffer. They were kept in an orbitary shaker at 100 rpm maintained at $37^{\circ} \mathrm{C}$. Aliquots of $2 \mathrm{ml}$ buffer were withdrawn at predefined time intervals and the medium was replaced with same volume of buffer. This study was carried out for $12 \mathrm{hrs}$, and the amount of drug release was estimated by determining the absorbance at $262 \mathrm{~nm}$ using Elico UV spectrophotometer [10].

\section{Results}

The obtained formulations were evaluated for the above mentioned parameters and the results are discussed as follows:

\section{Evaluation of niacin nanoparticles by desolvation technique}

- Niacin nanoparticles was carried out by Desolvation technique.

\section{Evaluation studies of niacin NPS}

- Product yield: The dried nanoparticles were weighed and calculated for product yield.

- Drug content: The drug content of the formulation was evaluated. It was observed that the NPs prepared by continuous addition method showed a drug content value i.e; 75.4\%.

- Entrapment efficiency: It was observed that the NPs prepared by continuous addition method showed a Entrapment efficiency value was found to be; $75.77 \%$.

- Loading capacity: It indicates the capacity of the polymer to load a drug. From the results it was found i.e, 25.44\%.

- Drug release studies: The drug release studies were performed by means of arbitrary shaker. In a time period of $12 \mathrm{hrs}$ $57.1 \%$ of the drug released from the formulation. 


\section{Discussion}

For desolvation technique

In this present study niacin nanoparticles were prepared by desolvation method by using BSA as polymer. using acetone as desolvating agent. This study was performed to determine the effect of continuous addition method on parameters such as entrapment efficiency, loading capacity, drug content was determined.

\section{Conclusion}

In the present study niacin nanoparticles were prepared by desolvation technique. In this technique a natural polymer such as Bovine Serum Albumin was selected for the study. In continuous addition method the desolvating agent was added at a rate of $1 \mathrm{ml}$ per min. The process parameters including $\mathrm{pH}$, stirring speed and stirring time were optimized.

The obtained nano formulation were studied for characterization parameters and evaluated for drug content, entrapment efficiency, loading capacity and in-vitro drug release.

\section{Acknowledgments}

The authors sincerely thank Dr. M. Sumakanth, The Principal, RBVRR Women's college of Pharmacy for providing access for library and databases. We also thank Mrs. Suvarna and Mrs. Sumalatha, for providing technical assistance.

\section{Bibliography}

1. N. K. Jain Advances in Controlled and Novel Drug Delivery, CBS Publishers and Distributers, New Delhi (2001): 408

2. Vyas SP and Khar RK. Targeted and Controlled drug delivey, Novel carrier systems, CBS publication (2002): 331-381.

3. Weber C., et al. "Desolvation process and surface characterisation of protein nanoparticles". International Journal of Pharmaceutics 194 (2000): 91-102.

4. K Sailaja P. Amareswar. Asian Journal Pharma Clinical Research 5(2012): 132-134.

5. Rahimnejad M., et al. "Production of biological nanoparticles from bovin Eserum albumin for drug delivery". African Journal of Biotechnology 5 (2005): 1918-1923.

6. Krishna Sailaja., et al. International Journal of Pharmacy and Pharmaceutics sciences 3 (2001): 1643-1647.

7. Kumar Ganesh., et al. International Journal of Pharmaceutical and Life Sciences 2 (2013): 202-229.

8. Abhilash M. International Journal of Pharma and Bio Sciences 1 (2011): 1-12.
9. Krishna Sailaja A and P Amareshwar. "Preparation of alginate nanoparticles by desovaltion technique using acetone as desolvating agent". Asian Journal of Pharmaceutical and Clinical Research 5 (2012).

10. Weber C., et al. "Desovation process and surface characterization of protein nanoparticles". International Journal of Pharmaceutics 194.1 (2000): 91-102.

\section{Volume 3 Issue 10 October 2019}

(C) All rights are reserved by Huda Syed and Abbaraju Krishna Sailaja. 